\title{
A Weak Limit Theorem for Numerical Approximation of Brownian Semi-stationary Processes
}

\author{
Mark Podolskij and Nopporn Thamrongrat
}

\begin{abstract}
In this paper we present a weak limit theorem for a numerical approximation of Brownian semi-stationary processes studied in [14]. In the original work of [14] the authors propose to use Fourier transformation to embed a given one dimensional (Lévy) Brownian semi-stationary process into a two-parameter stochastic field. For the latter they use a simple iteration procedure and study the strong approximation error of the resulting numerical scheme given that the volatility process is fully observed. In this work we present the corresponding weak limit theorem for the setting, where the volatility/drift process needs to be numerically simulated. In particular, weak approximation errors for smooth test functions can be obtained from our asymptotic theory.
\end{abstract}

Keywords Ambit fields • Brownian semi-stationary processes - Numerical schemes $\cdot$ Weak limit theorems

AMS 2010 Subject Classification: $60 \mathrm{~F} 05 \cdot 65 \mathrm{C} 30 \cdot 60 \mathrm{~F} 17$

\section{Introduction}

Recently, the mathematical theory of ambit fields has been intensively studied in the literature. Ambit fields is a class of spatio-temporal stochastic processes that has been originally introduced by Barndorff-Nielsen and Schmiegel in a series of papers [9-11] in the context of turbulence modelling, but which found manifold applications

\footnotetext{
M. Podolskij ( $ه)$

Department of Mathematics, University of Aarhus, Ny Munkegade 118,

8000 Aarhus C, Denmark

e-mail: mpodolskij@creates.au.dk

N. Thamrongrat

Department of Mathematics, Heidelberg University, Im Neuenheimer Feld 294,

69120 Heidelberg, Germany

e-mail: Thamrongrat@stud.uni-heidelberg.de 
in mathematical finance and biology among other sciences; see e.g. [4, 8]. In full generality they are described via the formula

$$
X_{t}(x)=\mu+\int_{A_{t}(x)} g(t, s, x, \xi) \sigma_{s}(\xi) L(\mathrm{~d} s, \mathrm{~d} \xi)+\int_{D_{t}(x)} q(t, s, x, \xi) a_{s}(\xi) \mathrm{d} s \mathrm{~d} \xi
$$

where $t$ typically denotes time while $x$ gives the position in space. Furthermore, $A_{t}(x)$ and $D_{t}(x)$ are ambit sets, $g$ and $q$ are deterministic weight functions, $\sigma$ represents the volatility or intermittency field, $a$ is a drift field and $L$ denotes a Lévy basis. We recall that a Lévy basis $L=\{L(B): B \in \mathscr{S}\}$, where $\mathscr{S}$ is a $\delta$-ring of an arbitrary non-empty set $S$ such that there exists an increasing sequence of sets $\left(S_{n}\right) \subset \mathscr{S}$ with $\cup_{n \in \mathbb{N}} S_{n}=S$, is an independently scattered random measure.

An important purely temporal subclass of ambit fields are the so called Lévy (Brownian) semi-stationary processes, which are defined as

$$
X_{t}=\mu+\int_{-\infty}^{t} g(t-s) \sigma_{s} L(\mathrm{~d} s)+\int_{-\infty}^{t} q(t-s) a_{s} \mathrm{~d} s,
$$

where now $L$ is a two-sided one dimensional Lévy (Brownian) motion and the ambit sets are given via $A_{t}=D_{t}=(-\infty, t)$. The notion of a semi-stationary process refers to the fact that the process $\left(X_{t}\right)_{t \in \mathbb{R}}$ is stationary whenever $\left(a_{t}, \sigma_{t}\right)_{t \in \mathbb{R}}$ is stationary and independent of $\left(L_{t}\right)_{t \in \mathbb{R}}$. In the past years stochastic analysis, probabilistic properties and statistical inference for Lévy semi-stationary processes have been studied in numerous papers. We refer to [2, 3, 6, 7, 11, 12, 15, 17, 20, 25] for the mathematical theory as well as to $[5,26]$ for a recent survey on theory of ambit fields and their applications.

For practical applications in sciences numerical approximation of Lévy (Brownian) semi-stationary processes, or, more generally, of ambit fields, is an important issue. We remark that due to a moving average structure of a Lévy semi-stationary process (cf. (2)) there exists no simple iterative Euler type approximation scheme. For this reason the authors of $[13,14]$ have proposed two different embedding strategies to come up with a numerical simulation. The first idea is based on the embedding of a Lévy semi-stationary process into a certain two-parameter stochastic partial differential equation. The second one is based upon a Fourier method, which again interprets a given Lévy semi-stationary process as a realization of a two-parameter stochastic field. We refer to the PhD thesis of Eyjolfsson [18] for a detailed analysis of both methods and their applications to modeling energy markets. We would also like to mention a very recent work [16], which investigates numerical simulations of spatio-temporal ambit fields.

The aim of this paper is to study the weak limit theory of the numerical scheme associated with the Fourier method proposed in [14, 18]. In the original work [14] the authors have discussed the strong approximation error (in the $L^{2}$ sense) of the numerical scheme for Lévy semi-stationary processes, where the volatility process $\left(\sigma_{t}\right)_{t \in \mathbb{R}}$ is assumed to be observed. We complement their study by analyzing the weak limit of the error process in the framework of Brownian semi-stationary processes, 
where the drift and the volatility processes need to be numerically simulated. This obviously gives a more precise assessment of the numerical error associated with the Fourier method.

The paper is organised as follows. In Sect. 2 we describe the Fourier approximation scheme for Brownian semi-stationary processes and present the main results on strong approximation error derived in $[14,18]$. Section 3 is devoted to a weak limit theorem associated with a slight modification of the Fourier method.

\section{Basic Assumptions and Fourier Approximation Scheme}

We start with a complete filtered probability space $\left(\Omega, \mathscr{F},(\mathscr{F})_{t \in \mathbb{R}}, \mathbb{P}\right)$, on which all processes are defined. We consider a Brownian semi-stationary process of the form

$$
X_{t}=\mu+\int_{-\infty}^{t} g(t-s) \sigma_{s} W(\mathrm{~d} s)+\int_{-\infty}^{t} q(t-s) a_{s} \mathrm{~d} s,
$$

where $g$ and $q$ are deterministic kernels, $\left(a_{t}\right)_{t \in \mathbb{R}}$ and $\left(\sigma_{t}\right)_{t \in \mathbb{R}}$ are adapted càdlàg processes, and $W$ is a two sided Brownian motion. To guarantee the finiteness of the first integral appearing in (3), we assume throughout the paper that

$$
\int_{-\infty}^{t} g^{2}(t-s) \sigma_{s}^{2} \mathrm{~d} s<\infty \quad \text { almost surely }
$$

for all $t \in \mathbb{R}$. When $\left(\sigma_{t}\right)_{t \in \mathbb{R}}$ is a square integrable stationary process, the above condition holds if $g \in L^{2}\left(\mathbb{R}_{\geq 0}\right)$. The presence of the drift process $\left(a_{t}\right)_{t \in \mathbb{R}}$ will be essentially ignored in this section.

Now, we describe the Fourier approximation method introduced in [14, 18] applied to the framework of Brownian semi-stationary processes. We start with the following assumptions on kernels involved in the description (3):

\section{Assumption (A):}

(i) The kernel functions $g$ and $q$ have bounded support contained in $[0, \tau]$ for some $\tau>0$.

(ii) $g, q \in C\left(\mathbb{R}_{\geq 0}\right)$.

In some cases these conditions are rather restrictive. We will give remarks on them below. For any given $\lambda>0$, we define

$$
h(x):=g(|x|) \text { and } h_{\lambda}(x):=h(x) \exp (\lambda|x|) .
$$


Notice that $g=h$ on $[0, \tau]$. We introduce the Fourier transform of $h_{\lambda}$ via

$$
\widehat{h}_{\lambda}(y):=\int_{\mathbb{R}} h_{\lambda}(x) \exp (-i x y) \mathrm{d} x .
$$

Furthermore, if we assume that $\widehat{h}_{\lambda} \in L^{1}(\mathbb{R})$, the inverse Fourier transform exists and we obtain the identity

$$
h(x)=\frac{\exp (-\lambda|x|)}{2 \pi} \int_{\mathbb{R}} \widehat{h}_{\lambda}(y) \exp (i x y) \mathrm{d} y .
$$

Since the Fourier transform maps $L^{1}(\mathbb{R})$ functions into the space of continuous functions, we require that $h \in C(\mathbb{R})$. This fact explains the Assumption (A)(ii) for the kernel function $g$. Since $h$ is an even function, for a given number $N \in \mathbb{N}$, we deduce an approximation of $h$ via

$$
h(x) \approx h_{N}(x):=\exp (-\lambda|x|)\left(\frac{b_{0}}{2}+\sum_{k=1}^{N} b_{k} \cos \left(\frac{k \pi x}{\tau}\right)\right)
$$

with

$$
b_{k}=\frac{\widehat{h}_{\lambda}(k \pi / \tau)}{\tau} \text {. }
$$

Obviously, the above approximation is an $L^{2}$-projection onto the linear subspace generated by orthogonal functions $\{\cos (k \pi x / \tau), \sin (k \pi x / \tau)\}_{k=0}^{N}$, hence we deal with a classical Fourier expansion of the function $h$ (recall that the function $h$ is even by definition, thus the sinus terms do not appear at (6)). Now, the basic idea of the numerical approximation method proposed in $[14,18]$ is based upon the following relationship:

$$
\begin{aligned}
\int_{u}^{t} g(t-s) \sigma_{s} W(\mathrm{~d} s) & \approx \int_{u}^{t} h_{N}(t-s) \sigma_{s} W(\mathrm{~d} s) \\
& =\int_{u}^{t} \exp (-\lambda(t-s))\left\{\frac{b_{0}}{2}+\sum_{k=1}^{N} b_{k} \cos \left(\frac{k \pi(t-s)}{\tau}\right)\right\} \sigma_{s} W(\mathrm{~d} s) \\
& =\frac{b_{0}}{2} \widehat{X}_{\lambda, u}(t, 0)+\operatorname{Re} \sum_{k=1}^{N} b_{k} \widehat{X}_{\lambda, u}\left(t, \frac{k \pi}{\tau}\right)
\end{aligned}
$$

where the complex valued stochastic field $\widehat{X}_{\lambda, u}(t, y)$ is defined via

$$
\widehat{X}_{\lambda, u}(t, y):=\int_{u}^{t} \exp \{(-\lambda+i y)(t-s)\} \sigma_{s} W(\mathrm{~d} s)
$$


and $u \in[t-\tau, t]$. In a second step, for a $\delta>0$ small, we observe the approximation

$$
\begin{aligned}
\widehat{X}_{\lambda, u}(t+\delta, y) & =\exp \{(-\lambda+i y) \delta\}\left(\widehat{X}_{\lambda, u}(t, y)+\int_{t}^{t+\delta} \exp \{(-\lambda+i y)(t-s)\} \sigma_{s} W(\mathrm{~d} s)\right) \\
& \approx \exp \{(-\lambda+i y) \delta\}\left(\widehat{X}_{\lambda, u}(t, y)+\sigma_{t}\left(W_{t+\delta}-W_{t}\right)\right) .
\end{aligned}
$$

Hence, we obtain a simple iterative scheme for simulating the stochastic field $\widehat{X}_{\lambda, u}(t, y)$ in the variable $t$. Assume for the moment that the drift process $a$ is zero and we wish to simulate the trajectory of $X_{t_{0}}, \ldots, X_{t_{M}}$ given the information available at time $t_{0}$. Then, the numerical simulation procedure is as follows:

(a) Simulate the independent increments $W_{t_{i}}-W_{t_{i-1}} \sim \mathscr{N}\left(0, t_{i}-t_{i-1}\right)$ for $i=$ $1, \ldots, M$.

(b) For each $i=1, \ldots, M$ and $k=0, \ldots, N$, simulate $\widehat{X}_{\lambda, u}\left(t_{i}, k \pi / \tau\right)$ from $\widehat{X}_{\lambda, u}\left(t_{i-1}, k \pi / \tau\right), W_{t_{i}}-W_{t_{i-1}}$ and $\sigma_{t_{i-1}}$ by using (10).

(c) Simulate $X_{t_{i}}$ applying steps (a), (b) and (8) (with $u=t_{0}$ ).

Let us explain some properties of the proposed numerical scheme. First of all, there are two approximation errors, where the first one ( $N$ scale) is coming from the Fourier transformation at (6) and the second one ( $M$ scale) is coming from the discretization error obtained at (10).

It is important to understand the meaning of knowing the information about the involved processes up to time $t_{0}$. When the stochastic model for the process $\left(\sigma_{t}\right)_{t \in \mathbb{R}}$ is uncoupled with $\left(X_{t}\right)_{t \in \mathbb{R}}$, then we may use $u=t-\tau$ at (8). Indeed, in typical applications such as turbulence and finance this is the case: $\left(\sigma_{t}\right)_{t \in \mathbb{R}}$ is usually modeled via a jump diffusion process driven by a Lévy process, which might be correlated with the Brownian motion $W$. However, when the process $\left(X_{t}\right)_{t \in \mathbb{R}}$ is itself of a diffusion type, i.e.

$$
X_{t}=\mu+\int_{t-\tau}^{t} g(t-s) \sigma\left(X_{s}\right) W(\mathrm{~d} s)+\int_{t-\tau}^{t} q(t-s) a\left(X_{s}\right) \mathrm{d} s
$$

it is in general impossible to simulate a trajectory of $\left(X_{t}\right)_{t \in \mathbb{R}}$, since for each value $t$ the knowledge of the path $\left(X_{u}\right)_{u \in(t-\tau, t)}$ is required to compute $X_{t}$. But, in case we do know the historical path, say, $\left(X_{u}\right)_{u \in[-\tau, 0]}$, the simulation of values $X_{t}, t \geq 0$, becomes possible.

The main advantage of the numerical scheme described above is that it separates the simulation of the stochastic ingredients $(\sigma$ and $W)$ and the approximation of the deterministic kernel $g$ (or $h$ ). In other words, the stochastic field $\widehat{X}_{\lambda, u}(t, y)$ is simulated via a simple recursive scheme without using the knowledge of $g$, while the kernel $g$ is approximated via the Fourier transform at (6). This is in contrast to a straightforward discretization scheme

$$
\int_{t_{0}}^{t_{j}} g(t-s) \sigma_{s} W(\mathrm{~d} s) \approx \sum_{i=1}^{j-1} g\left(t_{j}-t_{i}\right) \sigma_{t_{i}}\left(W_{t_{i+1}}-W_{t_{i}}\right)
$$


This numerical property is useful when considering a whole family of kernel functions $\left(g_{\theta}\right)_{\theta \in \Theta}$, since for any resulting model $X_{t}(\theta)$ only one realization of the stochastic field $\widehat{X}_{\lambda, u}(t, y)$ needs to be simulated. This can be obviously useful for the simulation of parametric Brownian semi-stationary processes.

We may now assess the strong approximation error of the proposed numerical scheme. We start with the analysis of the error associated with the approximation of the deterministic kernel $g$ by the function $h_{N}$. We assume for the moment that the volatility process $\left(\sigma_{t}\right)_{t \in \mathbb{R}}$ is square integrable with bounded second moment. Then a straightforward computation (see e.g. [14, Eq. (4.5)]) implies that

$\mathbb{E}\left[\left(\int_{t_{0}}^{t}\left\{g(t-s)-h_{N}(t-s)\right\} \sigma_{s} W(\mathrm{~d} s)\right)^{2}\right] \leq C \frac{1-\exp \left\{-2 \lambda\left(t-t_{0}\right)\right\}}{\lambda}\left(\sum_{k=N+1}^{\infty}\left|b_{k}\right|\right)^{2}$,

where $C$ is a positive constant and the Fourier coefficients $b_{k}$ have been defined at (7). We remark that $\left(1-\exp \left\{-2 \lambda\left(t-t_{0}\right)\right\}\right) / \lambda \rightarrow 2\left(t-t_{0}\right)$ as $\lambda \rightarrow 0$, while $\left(1-\exp \left\{-2 \lambda\left(t-t_{0}\right)\right\}\right) / \lambda \sim \lambda^{-1}$ as $\lambda \rightarrow \infty$. Thus, it is preferable to choose the parameter $\lambda>0$ large.

Remark 1 A standard model for the kernel function $g$ in the context of turbulence is given via

$$
g(x)=x^{\alpha} \exp (-\bar{\lambda} x)
$$

with $\bar{\lambda}>0$ and $\alpha>-1 / 2$. Obviously, this function has unbounded support and for the values $\alpha \in(-1 / 2,0)$ it is also discontinuous at 0 , hence it violates the statement of the Assumption (A). However, one can easily construct an approximating function $g_{\varepsilon}^{T}$, which coincides with $g$ on the interval $[\varepsilon, T]$ and satisfies the Assumption (A). Assuming again the boundedness of the second moment of the process $\left(\sigma_{t}\right)_{t \in \mathbb{R}}$, the approximation error is controlled via

$$
\mathbb{E}\left[\left(\int_{-\infty}^{t}\left\{g(t-s)-g_{\varepsilon}^{T}(t-s)\right\} \sigma_{s} W(\mathrm{~d} s)\right)^{2}\right] \leq C\left\|g-g_{\varepsilon}^{T}\right\|_{L^{2}((0, \varepsilon) \cup(T, \infty))}^{2}
$$

Such error can be made arbitrary small by choosing $\varepsilon$ small and $T$ large. Clearly, this is a rather general approach, which is not particularly related to a given class of kernel functions $g$. In a second step one would apply the Fourier approximation method described above to the function $g_{\varepsilon}^{T}$. At his stage it is important to note that the parameter $\lambda>0$ introduced at (5) is naturally restricted through the condition $\lambda<\bar{\lambda}$; otherwise the kernel $h_{\lambda}$ would have an explosive behaviour at $\infty$. Thus, the approximation error discussed at (11) cannot be made arbitrarily small in $\lambda$.

Remark 2 The Fourier coefficients $b_{k}$ can be further approximated under stronger conditions on the function $h$, which helps to obtain an explicit bound at (11). More 
specifically, when $h \in C^{2 n}(\mathbb{R})$ and $h_{\lambda}^{(2 j-1)}(\tau)=0$ for all $j=1, \ldots, n$, then it holds that

$$
\left|b_{k}\right| \leq C k^{-2 n} \text {. }
$$

This follows by a repeated application of integration by parts formula (see [14, Proposition 4.1] for a detailed exposition). In fact, the original work [14] defines another type of smooth interpolation functions $h$, rather than the mere identity $h(x)=$ $g(|x|)$, to achieve that the relationship $h_{\lambda}^{(2 j-1)}(\tau)=0$ holds for all $j=1, \ldots, n$ and some $n \in \mathbb{N}$.

Now, let us turn our attention to the discretization error introduced at (10). We assume that $t_{0}<\cdots<t_{M}$ is an equidistant grid with $t_{i}-t_{i-1}=\Delta t$. According to (10) the random variable

$$
\eta_{j}(y):=\sum_{i=1}^{j} \exp \{(-\lambda+i y)(j+1-i) \Delta t\} \sigma_{t_{i-1}}\left(W_{t_{i}}-W_{t_{i-1}}\right)
$$

is an approximation of $\widehat{X}_{\lambda, t_{0}}\left(t_{j}, y\right)$ for any $y \in \mathbb{R}$ whenever the drift process $a$ is assumed to be absent. When $\left(\sigma_{t}\right)_{t \in \mathbb{R}}$ is a weak sense stationary process, a straightforward computation proves that

$$
\mathbb{E}\left[\left|\widehat{X}_{\lambda, t_{0}}\left(t_{j}, y\right)-\eta_{j}(y)\right|^{2}\right] \leq C\left(t_{j}-t_{0}\right)\left(\left(\lambda^{2}+y^{2}\right)(\Delta t)^{2}+\mathbb{E}\left[\left|\sigma_{t_{1}}-\sigma_{t_{0}}\right|^{2}\right]\right) .
$$

We refer to [14, Lemma 4.2] for a detailed proof.

Remark 3 Assume that the process $\left(\sigma_{t}\right)_{t \in \mathbb{R}}$ is a continuous stationary Itô semimartingale, i.e.

$$
\mathrm{d} \sigma_{t}=\widetilde{a}_{t} \mathrm{~d} t+\widetilde{\sigma}_{t} \mathrm{~d} B_{t}
$$

where $B$ is a Brownian motion and $\left(\widetilde{a}_{t}\right)_{t \in \mathbb{R}},\left(\widetilde{\sigma}_{t}\right)_{t \in \mathbb{R}}$ are stochastic processes with bounded second moment. Then the Itô isometry implies that

$$
\mathbb{E}\left[\left|\sigma_{t_{1}}-\sigma_{t_{0}}\right|^{2}\right] \leq C \Delta t
$$

Hence, in this setting $\Delta t$ becomes the dominating term in the approximation error (13).

Combining the estimates at (11) and (13), we obtain the strong approximation error of the proposed Fourier method, which is the main result of [14] (see Propositions 4.1 and 4.3 therein).

Proposition 1 Let $t_{0}<\cdots<t_{M}$ be an equidistant grid with $t_{i}-t_{i-1}=\Delta t$. Assume that condition $(A)$ holds and $\left(\sigma_{t}\right)_{t \in \mathbb{R}}$ is a weak sense stationary process. Then the $L^{2}$ approximation error associated with the Fourier type numerical scheme is given via 


$$
\begin{aligned}
& \mathbb{E}\left[\left|\int_{t_{0}}^{t_{j}} g\left(t_{j}-s\right) \sigma_{s} W(d s)-\left(\frac{b_{0}}{2} \eta_{j}(0)+\sum_{k=1}^{N} b_{k} \eta_{j}\left(\frac{k \pi}{\tau}\right)\right)\right|^{2}\right] \\
& \leq C\left(\frac{1-\exp \left\{-2 \lambda\left(t-t_{0}\right)\right\}}{\lambda}\left(\sum_{k=N+1}^{\infty}\left|b_{k}\right|\right)^{2}\right. \\
& +\left(t_{j}-t_{0}\right)\left\{\lambda^{2}\left(\frac{\left|b_{0}\right|}{2}+\sum_{k=1}^{N}\left|b_{k}\right|\right)^{2}(\Delta t)^{2}+\left(\frac{\pi}{\tau}\right)^{2}\left(\sum_{k=1}^{N} k\left|b_{k}\right|\right)^{2}(\Delta t)^{2}\right. \\
& \left.\left.+\left(\frac{\left|b_{0}\right|}{2}+\sum_{k=1}^{N}\left|b_{k}\right|\right)^{2} \mathbb{E}\left[\left|\sigma_{t_{1}}-\sigma_{t_{0}}\right|^{2}\right]\right\}\right)
\end{aligned}
$$

for a positive constant $C$.

\section{A Weak Limit Theorem for the Fourier Approximation Scheme}

As we mentioned earlier, the Fourier approximation scheme investigated in $[14,18]$ basically ignored the need of simulating the volatility process $\left(\sigma_{t}\right)_{t \in \mathbb{R}}$ in practical applications (the same holds for the drift process $\left(a_{t}\right)_{t \in \mathbb{R}}$ ). As in the previous section we fix a time $t_{0}$ and assume the knowledge of all processes involved up to that time. Here we propose a numerical scheme for simulating the path $\left(X_{t}\right)_{t \in\left[t_{0}, T\right]}$ for a given terminal time $T>t_{0}$, which is a slightly modified version of the original Fourier approach. We recall the imposed condition (A), in particular, the weight functions $g$ and $q$ are assumed to have bounded support contained in $[0, \tau]$. First of all, we assume that we have càdlàg estimators $\left(a_{t}^{M}, \sigma_{t}^{M}\right)_{t \in\left[t_{0}, T\right]}$ of the stochastic process $\left(a_{t}, \sigma_{t}\right)_{t \in\left[t_{0}, T\right]}$ and the convergence rate $v_{M} \rightarrow \infty$ as $M \rightarrow \infty$ such that the following functional stable convergence holds:

$$
v_{M}\left(a^{M}-a, \sigma^{M}-\sigma\right) \stackrel{d_{s t}}{\longrightarrow} U=\left(U^{1}, U^{2}\right) \quad \text { on } D^{2}\left(\left[t_{0}, T\right]\right),
$$

where the convergence is on the space of bivariate càdlàg functions defined on $\left[t_{0}, T\right]$ equipped with the Skorohod topology $D^{2}\left(\left[t_{0}, T\right]\right)$. Let us briefly recall the notion of stable convergence, which is originally due to Rényi [27]. We say that a sequence of random variables $Y^{n}$ with values in a Polish space $(E, \mathscr{E})$ converges stably in law to $Y$, where $Y$ is defined on an extension $\left(\Omega^{\prime}, \mathscr{F}^{\prime}, \mathbb{P}^{\prime}\right)$ of the original probability $(\Omega, \mathscr{F}, \mathbb{P})$ if and only if

$$
\lim _{n \rightarrow \infty} \mathbb{E}\left[f\left(Y^{n}\right) Z\right]=\mathbb{E}^{\prime}[f(Y) Z]
$$


for any bounded and continuous function $f$ and any bounded $\mathscr{F}$-measurable random variable $Z$. In this case we write $\left(Y^{n} \stackrel{d_{s t}}{\longrightarrow} Y\right)$ ). In the following we will deal with the space of càdlàg processes equipped with the Skorohod topology or with the space of continuous processes equipped with the uniform topology. We refer to $[1,23]$ or [27] for a detailed study of stable convergence. Note that stable convergence is a stronger mode of convergence than weak convergence, but it is weaker than uniform convergence in probability.

We remark that the estimators $\left(a_{t}^{M}\right)_{t \in\left[t_{0}, T\right]}$ and $\left(\sigma_{t}^{M}\right)_{t \in\left[t_{0}, T\right]}$ might have a different effective convergence rate. In this case we will have either $U_{1} \equiv 0$ or $U_{2} \equiv 0$.

Now, we basically follow the Fourier type approach, which refers to (5) and the definition of the function $\widehat{h}_{\lambda}$, described in the previous section, but we replace the Fourier transform approximation proposed at (6) by a Riemann sum approximation. More specifically, we introduce the approximation

$$
\begin{aligned}
h(x) & =\frac{\exp (-\lambda|x|)}{2 \pi} \int_{\mathbb{R}} \widehat{h}_{\lambda}(y) \exp (i x y) \mathrm{d} y \\
& \approx \widetilde{h}_{N}(x):=\frac{\exp (-\lambda|x|)}{\pi N} \sum_{k=0}^{c_{N}} \widehat{h}_{\lambda}\left(\frac{k}{N}\right) \cos \left(\frac{k x}{N}\right),
\end{aligned}
$$

where $c_{N}$ is a sequence of numbers in $\mathbb{N}$ satisfying $c_{N} / N \rightarrow \infty$ as $N \rightarrow \infty$. In the following we will also assume that the sequence $c_{N}$ additionally satisfies the condition

$$
N \int_{c_{N} / N}^{\infty}\left|\widehat{h}_{\lambda}(y)\right| \mathrm{d} y \rightarrow 0 \quad \text { as } N \rightarrow \infty .
$$

Clearly, such a sequence exists, since $\widehat{h}_{\lambda} \in L^{1}(\mathbb{R})$. When introducing the approximation at (16), we obviously obtain two types of error: The Riemann sum approximation error and tail approximation error. Condition (17) guarantees that the Riemann sum approximation error will dominate.

Remark 4 Under some stronger conditions the tail integral at (17) can be bounded from above explicitly. Assume that $h \in C^{2}([-\tau, \tau])$ such that $h^{\prime}(\tau)=0$ (cf. Remark 2). Then a repeated application of integration by parts formula implies the identity

$$
\widehat{h}_{\lambda}(y)=\int_{\mathbb{R}} h_{\lambda}(x) \cos (y x) \mathrm{d} x=-\frac{1}{y^{2}} \int_{-\tau}^{\tau} h_{\lambda}^{\prime \prime}(x) \cos (y x) \mathrm{d} x
$$

for any $y>0$. Thus, for any $u>0$, we deduce the inequality

$$
\int_{u}^{\infty}\left|\widehat{h}_{\lambda}(y)\right| \mathrm{d} y \leq C\left\|h^{\prime \prime}\right\|_{L^{1}} \int_{u}^{\infty} y^{-2} \mathrm{~d} y \leq C\left\|h^{\prime \prime}\right\|_{L^{1}} u^{-1} .
$$

Hence, condition (17) holds whenever $N^{2} / c_{N} \rightarrow 0$ as $N \rightarrow \infty$. 
Remark 5 We remark that the Fourier transform used at (6) comes from the $L^{2}$ theory. Thus, in contrast to the $L^{2}$-distance $\left\|h-h_{N}\right\|_{L^{2}}$, the limiting behaviour of a standardized version of $h(x)-h_{N}(x)$ is difficult to study pointwise. This is precisely the reason why we use the Riemann sum approximation instead, for which we will show the convergence of $N\left(h(x)-\widetilde{h}_{N}(x)\right)$.

If one can freely choose the simulation rates $N$ and $M$, the Fourier transform of (6) is numerically more preferable. According to the estimate (11) and the upper bound for the Fourier coefficient of Remark 2 applied for $n=1$, we readily deduce the rate $N^{-1}$ for the $L^{2}$-error approximation connected to (6). On the other hand, the effective sample size of the Riemann approximation at (16) is $c_{N}$. In the setting of the previous remark the overall Riemann approximation error is $\max \left(N^{-1}, N / c_{N}\right)$. Recalling that $c_{N} / N \rightarrow \infty$, the obtained rate is definitely slower than the one associated with Fourier approximation proposed at (6).

Nevertheless, as our aim is to precisely determine the asymptotics associated with the $N$ scale, we will discuss the Riemann approximation approach in the sequel. A statement about the Fourier transform (6) will be presented in Remark 8.

Now, we essentially proceed as in the steps (8)-(10). First of all, it holds that

$$
\begin{aligned}
\int_{u}^{t} g(t-s) \sigma_{s} W(\mathrm{~d} s) & \approx \int_{u}^{t} \widetilde{h}_{N}(t-s) \sigma_{s} W(\mathrm{~d} s) \\
& =\int_{u}^{t} \exp (-\lambda(t-s))\left\{\sum_{k=0}^{c_{N}} \widetilde{b}_{k} \cos \left(\frac{k(t-s)}{N}\right)\right\} \sigma_{s} W(\mathrm{~d} s) \\
& =\operatorname{Re} \sum_{k=0}^{c_{N}} \widetilde{b}_{k} \widehat{X}_{\lambda, u}\left(t, \frac{k}{N}\right)
\end{aligned}
$$

where the complex valued stochastic field $\widehat{X}_{\lambda, u}(t, y)$ is defined at (9) and $\widetilde{b}_{k}=$ $\widehat{h}_{\lambda}(k / N) /(\pi N)$. In a second step, for $\delta>0$, we obtain the approximation

$$
\begin{aligned}
\widehat{X}_{\lambda, u}(t+\delta, y) & =\exp \{(-\lambda+i y) \delta\}\left(\widehat{X}_{\lambda, u}(t, y)+\int_{t}^{t+\delta} \exp \{(-\lambda+i y)(t-s)\} \sigma_{s} W(\mathrm{~d} s)\right) \\
& \approx \exp \{(-\lambda+i y) \delta\}\left(\widehat{X}_{\lambda, u}(t, y)+\int_{t}^{t+\delta} \exp \{(-\lambda+i y)(t-s)\} \sigma_{s}^{M} W(\mathrm{~d} s)\right) .
\end{aligned}
$$

When the estimator $\sigma^{M}$ is assumed to be constant on intervals $\left[s_{i-1}, s_{i}\right), i=$ $1, \ldots, M$, the last integral at (19) can be easily simulated (cf. (10)). We remark that this approximation procedure slightly differs from (10) as now we leave the exponential term unchanged. 
In summary, given that the information up to time $t_{0}$ is available, we arrive at the simulated value

$$
X_{t}^{N, M}:=\int_{t_{0}}^{t} \widetilde{h}_{N}(t-s) \sigma_{s}^{M} W(\mathrm{~d} s)+\int_{t_{0}}^{t} q(t-s) a_{s}^{M} \mathrm{~d} s
$$

of the random variable

$$
X_{t}^{0}=\int_{t_{0}}^{t} g(t-s) \sigma_{s} W(\mathrm{~d} s)+\int_{t_{0}}^{t} q(t-s) a_{s} \mathrm{~d} s .
$$

Note that the drift part of the Brownian semi-stationary process $X$ is estimated in a direct manner, although other methods similar to the treatment of the Brownian part are possible. Now, we wish to study the asymptotic theory for the approximation error $X_{t}^{N, M}-X_{t}^{0}$. Our first result analyzes the limiting behaviour of the function $N\left(h(x)-\widetilde{h}_{N}(x)\right)$.

Lemma 1 Define the function $\psi_{N}(x):=N\left(h(x)-\widetilde{h}_{N}(x)\right)$. Let us assume that the condition

$$
\widehat{y h_{\lambda}(y)} \in L^{1}(\mathbb{R}), \quad \widehat{y^{2} h_{\lambda}(y)} \in L^{1}(\mathbb{R})
$$

holds. Then, under Assumption (A), (17) and (22), it holds that

$$
\psi_{N}(x) \rightarrow \psi(x)=-\frac{\widehat{h}_{\lambda}(0)}{2 \pi} \exp (-\lambda|x|) \quad \text { as } N \rightarrow \infty
$$

for any $x \in \mathbb{R}$. Furthermore, it holds that

$$
\sup _{N \in \mathbb{N}, x \in[0, T]}\left|\psi_{N}(x)\right| \leq C
$$

for any $T>0$.

Proof First, we recall a well known result from Fourier analysis (see e.g. [19, Theorem 8.22]): The condition (22) implies that

$$
\widehat{h}_{\lambda}^{\prime} \in L^{1}(\mathbb{R}), \quad \widehat{h}_{\lambda}^{\prime \prime} \in L^{1}(\mathbb{R})
$$

Now, observe the decomposition

$$
\begin{aligned}
\psi_{N}(x) & =\frac{N \exp (-\lambda|x|)}{\pi} \sum_{k=0}^{c_{N}} \int_{k / N}^{(k+1) / N}\left(\kappa_{x}(y)-\kappa_{x}\left(\frac{k}{N}\right)\right) \mathrm{d} y \\
& +\frac{N \exp (-\lambda|x|)}{\pi} \int_{\left(c_{N}+1\right) / N}^{\infty} \kappa_{x}(y) \mathrm{d} y \\
& =\frac{N \exp (-\lambda|x|)}{\pi} \sum_{k=0}^{c_{N}} \int_{k / N}^{(k+1) / N}\left(\kappa_{x}(y)-\kappa_{x}\left(\frac{k}{N}\right)\right) \mathrm{d} y+o(1),
\end{aligned}
$$


where $\kappa_{x}(y)=\widehat{h}_{\lambda}(y) \cos (y x)$ and the approximation follows by the inequality $\left|\kappa_{x}(y)\right| \leq\left|\widehat{h}_{\lambda}(y)\right|$ and condition (17). Let us denote by $\kappa_{x}^{\prime}(y)$ the derivative of $\kappa_{x}(y)$ with respect to $y$. Since $\kappa_{x}^{\prime}(\cdot), \kappa_{x}^{\prime \prime}(\cdot) \in L^{1}\left(\mathbb{R}_{\geq 0}\right)$ because of (24), we deduce that

$$
\begin{aligned}
\psi_{N}(x) & =\frac{N \exp (-\lambda|x|)}{\pi} \sum_{k=0}^{c_{N}} \int_{k / N}^{(k+1) / N} \kappa_{x}^{\prime}\left(\frac{k}{N}\right)\left(y-\frac{k}{N}\right) \mathrm{d} y+o(1) \\
& =\frac{\exp (-\lambda|x|)}{2 \pi N} \sum_{k=0}^{c_{N}} \kappa_{x}^{\prime}\left(\frac{k}{N}\right)+o(1) \\
& \rightarrow \frac{\exp (-\lambda|x|)}{2 \pi} \int_{0}^{\infty} \kappa_{x}^{\prime}(y) \mathrm{d} y \quad \text { as } N \rightarrow \infty .
\end{aligned}
$$

But, since $\widehat{h}_{\lambda}$ vanishes at infinity, we readily obtain that

$$
\int_{0}^{\infty} \kappa_{x}^{\prime}(y) \mathrm{d} y=-\widehat{h}_{\lambda}(0)
$$

In order to prove the second assertion of the lemma, we observe the inequality

$$
\left|\psi_{N}(x)\right| \leq \frac{\exp (-\lambda|x|)}{\pi} \sum_{k=0}^{c_{N}} \int_{k / N}^{(k+1) / N}\left|\kappa_{x}^{\prime}\left(\zeta_{k, N}(y)\right)\right| \mathrm{d} y+N \int_{c_{N} / N}^{\infty}\left|\widehat{h}_{\lambda}(y)\right| \mathrm{d} y
$$

where $\zeta_{k, N}(y)$ is a certain value with $\zeta_{k, N}(y) \in(k / N, y)$. Clearly, the second term in the above approximation is bounded in $N$, since it converges to 0 . On the other hand, we have that $\left|\kappa_{x}^{\prime}(y)\right| \leq|x|\left|\widehat{h}_{\lambda}(y)\right|+\left|\widehat{h}_{\lambda}^{\prime}(y)\right|$, and since $\widehat{h}_{\lambda}, \widehat{h}_{\lambda}^{\prime} \in L^{1}\left(\mathbb{R}_{\geq 0}\right)$, we readily deduce that

$$
\sup _{N \in \mathbb{N}, x \in[0, T]}\left|\psi_{N}(x)\right| \leq C .
$$

This completes the proof of the lemma.

At this stage we need a further condition on the kernel function $g$ to prove tightness later.

\section{Assumption (B):}

(i) The kernel function $g$ has the form

$$
g(x)=x^{\alpha} f(x)
$$

for some $\alpha \geq 0$ and function $f$ satisfying $f(0) \neq 0$.

(ii) $f \in C^{1}\left(\mathbb{R}_{\geq 0}\right)$ has bounded support contained in $[0, \tau]$. 
Notice that the assumption $\alpha \geq 0$ is in accordance with the condition (A)(ii). Assumption (B) implies the following approximation result:

$$
\int_{0}^{1}|g(x+\delta)-g(x)|^{4} \mathrm{~d} x \leq \begin{cases}C \delta^{4} & \alpha=0 \\ C \delta^{\min (4,4 \alpha+1)} & \alpha>0\end{cases}
$$

for $\delta \in[0, T]$. The case $\alpha=0$ is trivial, while the other one follows along the lines of the proof of [20, Lemma 4.1]. As a matter of fact, we also require a good estimate of the left side of (25) when the kernel $g$ is replaced by the function $\psi_{N}$ defined in Lemma 1. In the following we will assume that

$$
\sup _{N \in \mathbb{N}} \int_{0}^{1}\left|\psi_{N}(x+\delta)-\psi_{N}(x)\right|^{4} \mathrm{~d} x \leq C \delta^{1+\varepsilon}
$$

for some $\varepsilon>0$ and $\delta \in[0, T]$.

Remark 6 Unfortunately, we have not been able to show the statement of (26) under the mere assumption of, say, condition (B). Obviously, as in the case of function $g$, condition (26) would hold if

$$
\psi_{N}(x)=x^{\alpha} f_{N}(x)
$$

where $f_{N} \in C^{1}\left(\mathbb{R}_{\geq 0}\right)$ with uniformly bounded derivative in $N \in \mathbb{N}$ and $x$ in a compact interval. We can prove condition (26) explicitly when the function $g$ is differentiable. Assume that $y \widehat{h}_{\lambda}(y), y \widehat{h}_{\lambda}^{\prime}(y) \in L^{1}\left(\mathbb{R}_{\geq 0}\right)$ and $c_{N}$ is chosen in such a way that the condition

$$
N \int_{c_{N} / N}^{\infty}\left|y \widehat{h}_{\lambda}(y)\right| \mathrm{d} y \rightarrow 0 \quad \text { as } N \rightarrow \infty
$$

is satisfied. As in Lemma 1 we conclude that $\left(\left|\partial_{x} \partial_{y} \kappa_{x}(y)\right| \leq\left(\left|\widehat{h}_{\lambda}(y)\right|+\left|y x \widehat{h}_{\lambda}(y)\right|+\right.\right.$ $\left.\left|y \widehat{h}_{\lambda}^{\prime}(y)\right|\right)$ and, as in the proof of Lemma 1, we deduce that

$$
\begin{aligned}
\sup _{x \in[0, T]}\left|\psi_{N}^{\prime}(x)\right| \leq & C\left(N \int_{c_{N} / N}^{\infty}\left|y \widehat{h}_{\lambda}(y)\right| \mathrm{d} y+N \int_{c_{N} / N}^{\infty}\left|\widehat{h}_{\lambda}(y)\right| \mathrm{d} y\right. \\
& \left.+\sum_{k=0}^{c_{N}} \int_{k / N}^{(k+1) / N}\left|\partial_{y} \kappa_{x}\left(\zeta_{k, N}(y)\right)\right| \mathrm{d} y+\sum_{k=0}^{c_{N}} \int_{k / N}^{(k+1) / N}\left|\partial_{x} \partial_{y} \kappa_{x}\left(\widetilde{\zeta}_{k, N}(y)\right)\right| \mathrm{d} y\right)
\end{aligned}
$$

for certain values $\zeta_{k, N}(y), \widetilde{\zeta}_{k, N}(y)$ in the interval $(k / N, y)$. Then, due to our integrability conditions, we obtain

$$
\sup _{N \in \mathbb{N}, x \in[0, T]}\left|\psi_{N}^{\prime}(x)\right|<\infty
$$


Moreover, condition (26) is trivially satisfied due to mean value theorem. However, showing (26) under Assumption (B) seems to be a much harder problem for $\alpha \in$ $(0,1)$.

The next result is the main theorem of our paper.

Theorem 1 Assume that conditions (A), (B), (17), (22) and (26) hold, and the processes $\left(\sigma_{t}\right)_{t \in\left[t_{0}, T\right]}$ and $\left(\sigma_{t}^{M}\right)_{t \in\left[t_{0}, T\right]}$ has finite fourth moment with $\sup _{t \in\left[t_{0}, T\right]} \mathbb{E}\left[\sigma_{t}^{4}\right]$ $<\infty$ and $\sup _{t \in\left[t_{0}, T\right]} \sup _{M \in \mathbb{N}} \mathbb{E}\left[\left(\sigma_{t}^{M}\right)^{4}\right]<\infty$. We also assume that the process $U_{t}^{M}=v_{M}\left(\sigma_{t}^{M}-\sigma_{t}\right)$ satisfies

$$
\sup _{t \in\left[t_{0}, T\right]} \sup _{M \in \mathbb{N}} \mathbb{E}\left[\left(U_{t}^{M}\right)^{4}\right]<\infty
$$

Then we obtain the decomposition

$$
X_{t}^{N, M}-X_{t}^{0}=A_{t}^{N, M}+B_{t}^{M}
$$

such that

$$
N A^{N, M} \stackrel{\text { u.c.p. }}{\Longrightarrow} A=\frac{\widehat{h}_{\lambda}(0)}{2 \pi} \int_{t_{0}}^{.} \exp (-\lambda(\cdot-s)) \sigma_{s} W(d s) \quad \text { as } N, M \rightarrow \infty,
$$

where ucp convergence means that $\sup _{t \in\left[t_{0}, T\right]}\left|A_{t}^{N}-A_{t}\right| \stackrel{\mathbb{P}}{\longrightarrow} 0$, and

$$
v_{M} B^{M} \stackrel{d_{s t}}{\longrightarrow} B=\int_{t_{0}}^{\cdot} g(\cdot-s) U_{s}^{2} W(d s)+\int_{t_{0}}^{\cdot} q(\cdot-s) U_{s}^{1} d s \quad \text { as } M \rightarrow \infty,
$$

where the stable convergence holds on the space $C\left(\left[t_{0}, T\right]\right)$ equipped with the uniform topology.

Proof We start with the decomposition $X_{t}^{N, M}-X_{t}^{0}=A^{N, M}+B_{t}^{M}$, where

$$
\begin{aligned}
A_{t}^{N, M} & =\int_{t_{0}}^{t}\left\{\tilde{h}_{N}(t-s)-g(t-s)\right\} \sigma_{s}^{M} W(\mathrm{~d} s), \\
B_{t}^{M} & =\int_{t_{0}}^{t} g(t-s)\left\{\sigma_{s}^{M}-\sigma_{s}\right\} W(\mathrm{~d} s)+\int_{t_{0}}^{t} q(t-s)\left\{a_{s}^{M}-a_{s}\right\} \mathrm{d} s .
\end{aligned}
$$

We begin by proving the stable convergence in (29). Let us first recall a classical result about weak convergence of semimartingales (see [23, Theorem VI.6.22] or [24]): Let $\left(Y_{s}^{n}\right)_{s \in\left[t_{0}, T\right]}$ be a sequence of càdlàg processes such that $Y^{n} \stackrel{d_{s t}}{\longrightarrow} Y$ on $D\left(\left[t_{0}, T\right]\right)$ equipped with the Skorohod topology. Then we obtain the weak convergence

$$
\int_{t_{0}}^{\cdot} Y_{s}^{n} W(\mathrm{~d} s) \Longrightarrow \int_{t_{0}}^{\cdot} Y_{s} W(\mathrm{~d} s) \quad \text { on } C\left(\left[t_{0}, T\right]\right)
$$


equipped with the uniform topology. This theorem is an easy version of the general result, since the integrator $W$ does not depend on $n$ and hence automatically fulfills the P-UT property. The stable nature of the aforementioned weak convergence follows by joint convergence $\left(\int_{0}^{*} Y_{s}^{n} W(\mathrm{~d} s), Y^{n}, W\right) \Longrightarrow\left(\int_{0}^{\cdot} Y_{s} W(\mathrm{~d} s), Y, W\right)(\mathrm{cf}$. [24]). Hence, we deduce that

$$
\int_{0}^{\cdot} Y_{s}^{n} W(\mathrm{~d} s) \stackrel{d_{s t}}{\longrightarrow} \int_{0}^{\cdot} Y_{s} W(\mathrm{~d} s) \quad \text { on } C\left(\left[t_{0}, T\right]\right)
$$

equipped with the uniform topology. It is important to note that this result can not be directly applied to the process $B_{t}^{M}$, since this process is not a semimartingale in general. Thus, we will prove the stable convergence (29) by showing the stable convergence of finite dimensional distributions and tightness.

We fix $u_{1}, \ldots, u_{k} \in\left[t_{0}, T\right]$. Due to the condition (15), the finite dimensional version of (30) and continuous mapping theorem for stable convergence, we conclude the joint stable convergence

$$
\begin{aligned}
& \left(\left\{v_{M} \int_{t_{0}}^{u_{j}} g\left(u_{j}-s\right)\left\{\sigma_{s}^{M}-\sigma_{s}\right\} W(\mathrm{~d} s)\right\}_{j=1, \ldots, k}, v_{M} \int_{t_{0}} q(\cdot-s)\left\{a_{s}^{M}-a_{s}\right\} \mathrm{d} s\right) \\
& \stackrel{d_{s t}}{\longrightarrow}\left(\left\{\int_{t_{0}}^{u_{j}} g\left(u_{j}-s\right) U_{s}^{2} W(\mathrm{~d} s)\right\}_{j=1, \ldots, k}, \int_{t_{0}} q(\cdot-s) U_{s}^{1} \mathrm{~d} s\right)
\end{aligned}
$$

as $M \rightarrow \infty$. Here we remark that the stable convergence for the second component indeed holds, since the mapping $F: C\left(\left[t_{0}, \tau\right]\right) \times D\left(\left[t_{0}, T\right]\right) \rightarrow C\left(\left[t_{0}, T\right]\right)$, $F(q, a)=\int_{t_{0}}^{\cdot} q(\cdot-s) a_{s} \mathrm{~d} s$ is continuous. Hence, we are left with proving tightness for the first component of the process $B_{t}^{M}$. We fix $u, t \in\left[t_{0}, T\right]$ with $t>u$ and observe the decomposition

$$
\begin{aligned}
& v_{M}\left(\int_{t_{0}}^{t} g(t-s)\left\{\sigma_{s}^{M}-\sigma_{s}\right\} W(\mathrm{~d} s)-\int_{t_{0}}^{u} g(u-s)\left\{\sigma_{s}^{M}-\sigma_{s}\right\} W(\mathrm{~d} s)\right) \\
& =v_{M}\left(\int_{u}^{t} g(t-s)\left\{\sigma_{s}^{M}-\sigma_{s}\right\} W(\mathrm{~d} s)\right. \\
& \left.+\int_{t_{0}}^{u}\{g(t-s)-g(u-s)\}\left\{\sigma_{s}^{M}-\sigma_{s}\right\} W(\mathrm{~d} s)\right):=R_{M}^{(1)}(t, u)+R_{M}^{(2)}(t, u) .
\end{aligned}
$$

Using Burkholder and Cauchy-Schwarz inequalities and (27), we have

$$
\mathbb{E}\left[\left|R_{M}^{(1)}(t, u)\right|^{4}\right] \leq C(t-u) \int_{u}^{t}|g(t-s)|^{4} \mathrm{~d} s .
$$

Thus, we conclude that

$$
\mathbb{E}\left[\left|R_{M}^{(1)}(t, u)\right|^{4}\right] \leq C(t-u)^{2}
$$


Now, using the same methods we conclude that

$$
\mathbb{E}\left[\left|R_{M}^{(2)}(t, u)\right|^{4}\right] \leq C \int_{t_{0}}^{u}|g(t-s)-g(u-s)|^{4} \mathrm{~d} s \leq C(t-u)^{\min (4,4 \alpha+1)},
$$

where we used the inequality (25). Thus, applying (32), (33) and the Kolmogorov's tightness criteria, we deduce the tightness of the first component of the process $B_{t}^{M}$. This completes the proof of (29).

Now, we show the pointwise convergence at (28). Recalling the notation from (23), we need to show that

$$
\int_{t_{0}}^{t}\left\{\psi_{N}(t-s)-\psi(t-s)\right\} \sigma_{s}^{M} W(\mathrm{~d} s) \stackrel{\mathbb{P}}{\longrightarrow} 0 \quad \text { as } N, M \rightarrow \infty
$$

for a fixed $t$. The Itô isometry immediately implies that

$$
\begin{aligned}
\sup _{M \in \mathbb{N}} \mathbb{E}\left[\left|\int_{t_{0}}^{t}\left\{\psi_{N}(t-s)-\psi(t-s)\right\} \sigma_{s}^{M} W(\mathrm{~d} s)\right|^{2}\right] & \leq C \int_{t_{0}}^{t}\left\{\psi_{N}(t-s)-\psi(t-s)\right\}^{2} \mathrm{~d} s \\
& \rightarrow 0 \quad \text { as } N \rightarrow \infty
\end{aligned}
$$

which follows by Lemma 1 and the dominated convergence theorem. Hence, we obtain pointwise convergence at (28). Since the limiting process $A$ is continuous, we now need to show that

$$
\sup _{N, M \in \mathbb{N}} \mathbb{E}\left[N^{4}\left(A_{t}^{N, M}-A_{u}^{N, M}\right)^{4}\right] \leq C(t-u)^{1+\varepsilon}
$$

for $t_{0}<u<t$, to conclude ucp convergence from pointwise convergence in probability. Applying the same methods as in (32), (33) we deduce the inequality

$$
\begin{aligned}
& \sup _{N, M \in \mathbb{N}} \mathbb{E}\left[N^{4}\left(A_{t}^{N, M}-A_{u}^{N, M}\right)^{4}\right] \\
& \leq C\left((t-u) \int_{u}^{t}\left|\psi_{N}(t-s)\right|^{4} \mathrm{~d} s+\int_{t_{0}}^{u}\left|\psi_{N}(t-s)-\psi_{N}(u-s)\right|^{4} \mathrm{~d} s\right) \\
& \leq C(t-u)^{1+\varepsilon},
\end{aligned}
$$

which follows by Lemma 1 and condition (26). This completes the proof of Theorem 1.

Remark 7 We remark that the stronger conditions (B) and (26) are not required to prove the finite dimensional version of convergence (28) and (29).

Theorem 1 immediately applies to the weak approximation error analysis. Assume for simplicity that $M=M(N)$ is chosen such that $v_{M} / N \rightarrow 1$, so that the Riemann sum approximation error and the simulation error from (15) are balanced. We consider a 
bounded test function $\varphi \in C^{1}(\mathbb{R})$ with bounded derivative. The mean value theorem implies the identity

$$
\varphi\left(X_{t}^{N, M}\right)-\varphi\left(X_{t}^{0}\right)=\varphi^{\prime}\left(\xi_{N, M}\right)\left(X_{t}^{N, M}-X_{t}^{0}\right),
$$

where $\xi_{N, M}$ is a random value between $X_{t}^{0}$ and $X_{t}^{N, M}$ with $\xi_{N, M} \stackrel{\mathbb{P}}{\longrightarrow} X_{t}^{0}$ as $N \rightarrow \infty$. By properties of stable convergence we deduce that $\left(\xi_{N, M}, N\left(X_{t}^{N, M}-X_{t}^{0}\right)\right) \stackrel{d_{s t}}{\longrightarrow}$ $\left(X_{t}^{0}, A_{t}+B_{t}\right)$. Hence, given the existence of the involved expectations, we conclude that

$$
\mathbb{E}\left[\varphi\left(X_{t}^{N, M}\right)\right]-\mathbb{E}\left[\varphi\left(X_{t}^{0}\right)\right]=N^{-1} \mathrm{e}^{\prime}\left[\varphi^{\prime}\left(X_{t}^{0}\right)\left(A_{t}+B_{t}\right)\right]+o\left(N^{-1}\right) .
$$

(Recall that the limit $A_{t}+B_{t}$ is defined on the extended probability space $\left.\left(\Omega^{\prime}, \mathscr{F}^{\prime}, \mathbb{P}^{\prime}\right)\right)$.

Remark 8 The results of Theorem 1 may also apply to the original Fourier approximated method proposed in $[14,18]$. Let us keep the notation of this section and still denote the approximated value of $X_{t}^{0}$ by $X_{t}^{N, M}$. Recalling the result of (11) (see also Remark 2) and assuming that $M=M(N)$ is chosen such that $\sum_{k=N+1}^{\infty}\left|b_{k}\right| \ll v_{M}$, we readily deduce that

$$
v_{M}\left(X_{t}^{N, M}-X_{t}^{0}\right) \stackrel{d_{s t}}{\longrightarrow} B_{t} .
$$

Remark 9 The results of Theorem 1 might transfer to the case of Lévy semistationary processes

$$
X_{t}=\mu+\int_{-\infty}^{t} g(t-s) \sigma_{s} L(\mathrm{~d} s)+\int_{-\infty}^{t} q(t-s) a_{s} \mathrm{~d} s
$$

under suitable moment assumptions on the driving Lévy motion $L$ (cf. [14]). However, when $L$ is e.g. a $\beta$-stable process with $\beta \in(0,2)$, it seems to be much harder to access the weak limit of the approximation error.

In the following we will present some examples of convergence at (15) to highlight the most prominent results. For simplicity we assume that $a \equiv 0$ in all cases.

Example 1 Let us consider a continuous diffusion model for the volatility process $\sigma$, i.e.

$$
\mathrm{d} \sigma_{t}=\widetilde{a}\left(\sigma_{t}\right) \mathrm{d} t+\widetilde{v}\left(\sigma_{t}\right) \mathrm{d} B_{t}, \quad \sigma_{t_{0}}=x_{0},
$$

where $B$ is a Brownian motion possibly correlated with $W$. We consider an equidistant partition $t_{0}=s_{0}<s_{1}<\cdots<s_{M}=T$ of the interval $\left[t_{0}, T\right]$ and define the continuous Euler approximation of $\sigma_{t}$ via

$$
\sigma_{t}^{M}=\sigma_{s_{k}}^{M}+\widetilde{a}\left(\sigma_{s_{k}}^{M}\right)\left(t-s_{k}\right)+\widetilde{v}\left(\sigma_{s_{k}}^{M}\right)\left(B_{t}-B_{s_{k}}\right), \quad t \in\left[s_{k}, s_{k+1}\right] .
$$


When the functions $\widetilde{a}$ and $\widetilde{v}$ are assumed to be globally Lipschitz and continuously differentiable, it holds that

$$
\sqrt{M}\left(\sigma^{M}-\sigma\right) \stackrel{d_{s t}}{\longrightarrow} U^{2} \quad \text { on } C\left(\left[t_{0}, T\right]\right),
$$

where $U^{2}$ is the unique solution of the stochastic differential equation

$$
\mathrm{d} U_{t}^{2}=\widetilde{a}^{\prime}\left(\sigma_{t}\right) U_{t}^{2} \mathrm{~d} t+\widetilde{v}^{\prime}\left(\sigma_{t}\right) U_{t}^{2} \mathrm{~d} B_{t}-\frac{1}{\sqrt{2}} \widetilde{v} \widetilde{v}^{\prime}\left(\sigma_{t}\right) \mathrm{d} W_{t}^{\prime},
$$

where $W^{\prime}$ is a new Brownian motion independent of $\mathscr{F}$. We refer to [22, Theorem 1.2] for a detailed treatment of this result.

Example 2 Let us now consider a discontinuous diffusion model for the volatility process $\sigma$, i.e.

$$
\mathrm{d} \sigma_{t}=\widetilde{v}\left(\sigma_{t-}\right) \mathrm{d} L_{t}, \quad \sigma_{t_{0}}=x_{0},
$$

where $L$ is a purely discontinuous Lévy process. In this framework we study the discretized Euler scheme given via

$$
\sigma_{s_{k+1}}^{M}=\widetilde{v}\left(\sigma_{s_{k}}^{M}\right)\left(L_{s_{k+1}}-L_{s_{k}}\right), \quad k=0, \ldots, M-1 .
$$

We define the process $U_{t}^{M}=\sigma_{[t M] / M}^{M}-\sigma_{[t M] / M}$. In [21] several classes of Lévy processes $L$ has been studied. For the sake of exposition we demonstrate the case of a symmetric $\beta$-stable Lévy process $L$ with $\beta \in(0,2)$. Let us assume that $\widetilde{v} \in C^{3}(\mathbb{R})$. Then, it holds that

$$
(M / \log (M))^{1 / \beta} U^{M} \stackrel{d_{s t}}{\longrightarrow} U^{2} \quad \text { on } D\left(\left[t_{0}, T\right]\right),
$$

where $U^{2}$ is the unique solution of the linear equation

$$
\mathrm{d} U_{t}^{2}=\widetilde{v}^{\prime}\left(\sigma_{t-}\right) U_{t-}^{2} \mathrm{~d} L_{t}-\widetilde{v} \widetilde{v}^{\prime}\left(\sigma_{t-}\right) \mathrm{d} L_{t}^{\prime}
$$

and $L^{\prime}$ is another symmetric $\beta$-stable Lévy process (with certain scaling parameter) independent of $\mathscr{F}$. We note that this result does not directly correspond to our condition (15) as the discretized process $\sigma_{[t M] / M}$ is used in the definition of $U_{t}^{M}$.

Acknowledgments Nopporn Thamrongrat gratefully acknowledges support by Deutsche Forschungsgemeinschaft through the Research Training Group RTG 1953.

Open Access This chapter is distributed under the terms of the Creative Commons Attribution Noncommercial License, which permits any noncommercial use, distribution, and reproduction in any medium, provided the original author(s) and source are credited. 


\section{References}

1. Aldous, D.J., Eagleson, G.K.: On mixing and stability of limit theorems. Ann. Probab. 6(2), 325-331 (1978)

2. Barndorff-Nielsen, O.E., Benth, F.E., Pedersen, J., Veraart, A.: On stochastic integration for volatility modulated Lévydriven Volterra processes. Stochast. Process. Appl. 124(1), 812-847 (2014)

3. Barndorff-Nielsen, O.E., Benth, F.E., Szozda, B.: On stochastic integration for volatility modulated Brownian-driven Volterra processes via white noise analysis. Infin. Dimens. Anal. Quantum Probab. Relat. Top. 17(2), 1450011 (2014)

4. Barndorff-Nielsen, O.E., Benth, F.E., Veraart, A.: Modelling energy spot prices by volatility modulated Lévy-driven Volterra processes. Bernoulli. 19(3), 803-845 (2013)

5. Barndorff-Nielsen, O.E., Benth, F.E., Veraart, A.: Recent advances in ambit stochastics with a view towards tempo-spatial stochastic volatility/intermittency. To appear in Banach Center Publications (2014)

6. Barndorff-Nielsen, O.E., Corcuera, J.M., Podolskij, M.: Multipower variation for Brownian semistationary processes. Bernoulli. 17(4), 1159-1194 (2011)

7. Barndorff-Nielsen, O.E., Corcuera, J.M., Podolskij, M.: Limit theorems for functionals of higher order differences of Brownian semi-stationary processes. In: Honor of Yuri Prokhorov, V., Shiryaev, A.N., Varadhan, S.R.S., Presman E.L. (eds.) Prokhorov and Contemporary Probability Theory, vol. 33, pp. 69-96. Springer (2013)

8. Barndorff-Nielsen, O.E., Jensen, E.B.V., Jónsdóttir, K.Y., Schmiegel, J.: Spatio-temporal modelling — with a view to biological growth. In: Finkenstdt, B., Held L., Isham, V. Statistical Methods for Spatio-Temporal Systems, pp. 47-75. Chapman and Hall/CRC, London (2007)

9. Barndorff-Nielsen, O.E., Schmiegel, J.: Ambit processes; with applications to turbulence and cancer growth. In: Benth, F.E., Nunno, G.D., Linstrøm, T., Øksendal, B., Zhang, T. (eds.). Stochastic Analysis and Applications. The Abel Symposium 2005, pp. 93-124. Springer, Heidelberg (2007)

10. Barndorff-Nielsen, O.E., Schmiegel, J.: Time change, volatility and turbulence. In: Sarychev, A., Shiryaev, A., Guerra, M., Grossinho, M.D.R. (eds.) Proceedings of the Workshop on Mathematical Control Theory and Finance, Lisbon 2007, pp. 29-53. Springer, Berlin (2008)

11. Barndorff-Nielsen, O.E., Schmiegel, J.: Brownian semistationary processes and volatility/intermittency. In: Albrecher, H., Runggaldier, W., Schachermayer, W. (eds.) Advanced Financial Modelling. Radon Series on Computational and Applied Mathematics, vol. 8, pp. $1-26$ (2009)

12. Basse-O'Connor, A., Lachièze-Rey, R., Podolskij, M.: Limit theorems for stationary increments Lévy driven moving average processes. Working paper (2015)

13. Benth, F.E., Eyjolfsson, H.: Simulation of volatility modulated Volterra processes using hyperbolic stochastic partial differential equations. To appear in Bernoulli (2015)

14. Benth, F.E., Eyjolfsson, H., Veraart, A.E.D.: Approximating Lévy semistationary processes via Fourier methods in the context of power markets. SIAM J. Financ. Math. 5(1), 71-98 (2014)

15. Benth, F.E., Süß, A.: Integration theory for infinite dimensional volatility modulated Volterra processes. Working paper. arXiv:1303.7143. To appear in Bernoulli (2013)

16. Chen, B., Chong, C., Klüppelberg, C.: Simulation of stochastic Volterra equations driven by spacetime Lévy noise. Working paper. arXiv:1501.01645 (2015)

17. Corcuera, J.M., Hedevang, E., Pakkanen, M., Podolskij, M.: Asymptotic theory for Brownian semi-stationary processes with application to turbulence. Stochastic Process. Appl. 123, 2552 2574 (2013)

18. Eyjolfsson, H.: Approximation methods for ambit fields applied to power markets. Ph.D. thesis, Department of Mathematics, University of Oslo (2013)

19. Folland, G.B.: Real analysis—-modern techniques and their applications. Wiley\&Sons, J. (1984)

20. Gärtner, K., Podolskij, M.: On non-standard limits of Brownian semi-stationary processes. Stochastic Process. Appl. 125(2), 653-677 (2015) 
21. Jacod, J.: The Euler scheme for Lévy driven stochastic differential equations: limit theorems. Ann. Probab. 32(3), 1830-1872 (2004)

22. Jacod, J., Protter, P.: Asymptotic error distributions for the Euler method for stochastic differential equations. Ann. Probab. 26(1), 267-307 (1998)

23. Jacod, J., Shiryaev, A.N.: Limit theorems for stochastic processes, $2 \mathrm{~d}$ edn. Springer, Berlin (2002)

24. Kurtz, T.G., Protter, P.: Weak limit theorems for stochastic integrals and stochastic differential equations. Ann. Probab. 19(3), 1035-1070 (1993)

25. Pakkanen, M.: Limit theorems for power variations of ambit fields driven by white noise. Stochastic Process. Appl. 124(5), 1942-1973 (2014)

26. Podolskij, M.: Ambit fields: survey and new challenges. In: Mena, R., Pardo, J.C., Rivero, V., Bravo, G.U. (eds.) To appear in Special Volume of XI Symposium of Probability and Stochastic Processes (2014)

27. Rényi, A.: On stable sequences of events. Sankhyā Ser. A. 25, 293-302 (1963) 